\title{
Degrees of monotonicity of spatial transformations
}

\author{
Bart Kuijpers \\ University of Antwerp*
}

\begin{abstract}
We consider spatial databases that can be defined in terms of polynomial inequalities, and we are interested in monotonic transformations of spatial databases.

We investigate a hierarchy of monotonicity classes of spatial transformations that is determined by the number of degrees of freedom of the transformations. The result of a monotonic transformation with $k$ degrees of freedom on a spatial database is completely determined by its result on subsets of cardinality at most $k$ of the spatial database. The result of a transformation in the largest class of the hierarchy on a spatial database is determined by its result on arbitrary large subsets of the database. The latter is the class of all the monotonic spatial transformations.

We give a sound and complete language for the monotonic spatial transformations that can be expressed in the relational calculus augmented with polynomial inequalities and that belong to a class with a finite number of degrees of freedom. In particular, we show that these transformations are finite unions of transformations that can be written in a particular conjunctive form. We also address the problem of finding sound and complete languages for monotonic transformations that are expressible in the calculus and have an infinite number of degrees of freedom. We show that Lyndon's theorem, which is known to fail in finite model theory, also fails in this setting: monotonic spatial transformations expressible in the calculus do not correspond to the transformations expressible by a positive formula.

We show that it is undecidable whether a query expressed in the relational calculus augmented with polynomial inequalities is a monotonic spatial transformation of a certain degree. On the other hand, various interesting properties (e.g., equivalence, genericity), which are undecidable for general spatial transformations expressible in the calculus, become decidable for monotonic spatial transformations of finite degree.
\end{abstract}

\section{Introduction and summary}

Spatial database systems $[1,7,10,11]$ are concerned with the representation and manipulation of data that have a geometrical or topological interpretation. The conceptual view of such a database is that of a possibly infinite set of points

\footnotetext{
* Address: UIA, Informatica, Universiteitsplein 1, B-2610 Antwerpen, Belgium. Email: kuijpers@uia.ua.ac.be
} 
in the real space. The framework of constraint databases, introduced by Kanellakis, Kuper, and Revesz [15], provides an elegant and powerful model of spatial databases [20]. In this setting, a spatial database is represented as a Boolean combination of polynomial equalities and inequalities. For example, the spatial database consisting of the set of points on the northern hemisphere together with the points on the equator of the unit sphere in the three-dimensional space $\mathbf{R}^{3}$ can be represented by the formula $x^{2}+y^{2}+z^{2}=1 \wedge z \geq 0$. The set of points in the real plane lying strictly above the parabola $y=x^{2}$ and strictly below the parabola $y=x^{2}+1 / 2$ is another example of a spatial database. This database is depicted in Figure 1.

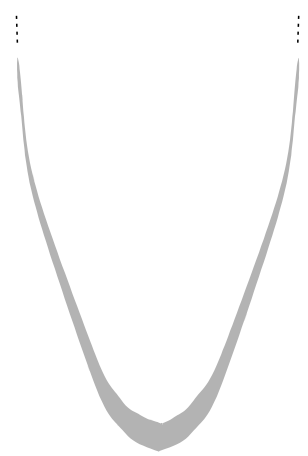

Fig. 1. A spatial database that consists of the points lying strictly between the parabola $y=x^{2}$ and the translated one $y=x^{2}+1 / 2$.

Several languages have been proposed to query and transform spatial databases in the constraint model. If, e.g., the relational calculus is extended with polynomial inequalities, a simple but effective query language is obtained [20]. The translation of a 2 -dimensional spatial database $S$ by the vector $(1,2)$, for instance, can be expressed in this language by the formula

$$
\left(\exists x^{\prime}\right)\left(\exists y^{\prime}\right)\left(S\left(x^{\prime}, y^{\prime}\right) \wedge x^{\prime}+1=x \wedge y^{\prime}+2=y\right) .
$$

The free variables $x$ and $y$ of this formula are the coordinates of the points in the result of the transformation. Although their variables range over the real numbers, such calculus expressions can be computed effectively $[6,8,22]$.

Also, various extensions of this calculus with recursion have been introduced and studied. In $[15,16,17]$, a spatial version of Datalog has been studied. In [13], computationally complete languages for spatial database queries and transformations were studied.

In this paper, we are especially interested in monotonic transformations of spatial databases. A transformation is monotone if it yields increasing outputs on 
increasing inputs. This interest is motivated by classes of transformations that frequently occur in spatial database practice $[1,7,10,11]$. The spatial transformation determined by the expression $(*)$ is monotone and it is exemplary for such a class. It works pointwise on the spatial data, i.e., its result on a spatial database is the union of the results of the transformation when applied to the individual points of the spatial database. Many other transformations, like rotations or projections, also satisfy this property. "Return the points within distance 1 from the input database" is another example of a pointwise spatial transformation. The result of these transformations is completely determined by their result on single points.

By adding one extra degree of freedom, we obtain a larger class of monotonic transformations whose results are determined by the results of the transformations on subsets with at most two elements. "Return the midpoints of each pair of different points in the input database" is a transformation with two degrees of freedom. It is not a pointwise transformation, however. The transformation of a spatial database into itself if its diameter is larger than 1 and into the empty set if it is not larger than 1 is another example.

Continuing in this way, every larger number of degrees of freedom gives rise to a larger class of monotonic transformations. The monotonic transformations with $k$ degrees of freedom are exactly the transformations that are monotonic and that are completely determined by their result on sets of cardinality at most $k$. An example of a monotonic transformations with $k$ degrees of freedom that is of practical importance is the transformation of a $(k-1)$-dimensional spatial database into its convex hull.

If the number of degrees of freedom is uncountably infinite, we obtain, as we will show, the set of all monotonic spatial transformations. We thus obtain a hierarchy of monotonicity classes of spatial transformations that is indexed by degrees of freedom (i.e., by cardinal numbers).

We syntactically describe sound and complete languages that capture the monotonic spatial transformations that are expressible in the relational calculus augmented with polynomial inequalities and that have a finite number of degrees of freedom. We show, more precisely, that these transformations are finite unions of transformations that can be written in a particular conjunctive form.

We also address the problem of finding sound and complete languages for monotonic transformations that are expressible in the calculus and that have an infinite (countable or uncountable) number of degrees of freedom. From model theory, Lyndon's theorem suggests that the set of transformations expressible by a positive calculus formula is likely to capture the set of all monotonic transformations expressible in the calculus. We show, however, that Lyndon's theorem, which is known to fail in finite model theory, also fails in our setting.

Finally, we show that it is undecidable whether a calculus formula expresses a monotonic spatial transformation of a certain degree. On the other hand, various interesting properties (e.g., equivalence, genericity), that are undecidable for general spatial database transformations, become decidable for monotonic spatial transformations that are expressible in the calculus and that have a finite 
number of degrees of freedom.

This paper is organized as follows. In Section 2, we give the definitions of spatial databases, spatial transformations and of monotonic transformations along with some basic properties of monotonic transformations. In Section 3, we present sound and complete languages for monotonic transformations of finite degree. In Section 4, we discuss complete languages for monotonic transformations of infinite degree. We give decidability and undecidability results in Section 5. We end the paper with a discussion in Section 6. We discuss alternative hierarchies of monotonicity classes and state some open problems concerning complete languages for monotonic transformations.

\section{Definitions and preliminaries}

In this section, we define spatial databases and spatial database transformations. Also, the notion of monotonic spatial transformation of degree $k$ is defined and illustrated.

\subsection{Spatial databases and spatial database transformations}

$\mathbf{R}$ denotes the set of the real numbers. So, $\mathbf{R}^{m}$ is the corresponding $m$-dimensional space, where $m$ is arbitrarily fixed.

Definition 1. A spatial database is a geometrical figure in $\mathbf{R}^{m}$ that can be defined as a Boolean combination of sets of the form $\left\{\left(x_{1}, \ldots, x_{m}\right) \mid p\left(x_{1}, \ldots, x_{m}\right)>\right.$ $0\}$, where $p\left(x_{1}, \ldots, x_{m}\right)$ is a polynomial with integer coefficients in the real variables $x_{1}, \ldots, x_{m}{ }^{2}$

The set $\left\{(x, y, z) \mid x^{2}+y^{2}+z^{2}=1 \wedge z \geq 0\right\}$, mentioned in the Introduction, is an example of a spatial database in $\mathbf{R}^{3}$. The polynomial equality $p=0$ can be seen as an abbreviation for $\neg(p>0) \wedge \neg(-p>0)$. The set $\{(x, y) \mid(y-$ $\left.\left.x^{2}\right)\left(x^{2}-y+1 / 2\right)>0\right\}$, also mentioned in the Introduction, is a spatial database in $\mathbf{R}^{2}$.

Definition 2. A spatial database transformation (or just transformation) is a function that maps every spatial database to a spatial database.

In this paper, we will use the relational calculus augmented with polynomial inequalities, the spatial calculus for short, as a language to express transformations. In this language, the result of a transformation $Q$ on an input spatial database $S$ is of the form

$$
Q(S)=\left\{\left(x_{1}, \ldots, x_{m}\right) \mid \varphi\left(x_{1}, \ldots, x_{m}, S\right)\right\},
$$

\footnotetext{
${ }^{2}$ Often, a spatial database is defined as a $n$-tuple of such geometrical figures (see, e.g., $[13,20])$. In order not to overload the notations further on, we have restricted ourselves to $n=1$. The results of this paper carry over straightforwardly to the more general situation.
} 
with $\varphi$ a formula built from the atomic formulas $S\left(y_{1}, \ldots, y_{m}\right)$ and $p\left(z_{1}, \ldots, z_{n}\right)>$ 0 , the logical connectives $\neg, \wedge, \vee$ and the quantifiers $\forall, \exists$. Here, $S$ is an $m$-ary relation name that represents the input spatial database and $p\left(z_{1}, \ldots, z_{n}\right)$ is a polynomial with integer coefficients and real variables $z_{1}, \ldots, z_{n}$.

Formula $(*)$ in the Introduction is an example of a spatial calculus formula that expresses a transformation in $\mathbf{R}^{2}$.

Further on, to economize on space and to improve readability, we will also use the vector notation in expressions. We use bold characters to denote vectors $\mathbf{x}$ in $\mathbf{R}^{m}$. Also, if $\mathbf{x} \in \mathbf{R}^{m}$, then we abbreviate, e.g., $\left(\exists x_{1}\right) \cdots\left(\exists x_{m}\right)\left(\varphi\left(x_{1}, \ldots, x_{m}\right)\right)$ by $(\exists \mathbf{x})(\varphi(\mathbf{x}))$.

\subsection{Monotonic transformations of degree $k$}

Let $\mathbf{N}$ denote the set of the natural numbers and let $|S|$ denote the cardinality of the set $S$.

Definition 3. A transformation $Q$ is called monotone if for all spatial databases $S$ and $S^{\prime}, S \subseteq S^{\prime}$ implies $Q(S) \subseteq Q\left(S^{\prime}\right)$.

It can easily be verified that $Q$ is monotone if and only if

$$
Q(S)=\bigcup_{S^{\prime} \subseteq S} Q\left(S^{\prime}\right)
$$

for all spatial databases $S$.

Definition 4. Let $k$ be a cardinal number less than or equal to $|\mathbf{R}|$. A monotonic spatial database transformation of degree $k$ is a spatial transformation that is monotone and that is completely determined by its result on sets of cardinality at most $k$.

We denote the set of all monotonic spatial database transformations of degree $k$ by $\mathcal{M}^{k}$ and the set of all monotonic transformations by $\mathcal{M}$. Before giving examples and counterexamples of monotonic transformations of degree $k$, we give some of their basic properties, the proofs of which follow straightforwardly from the above observation and the subsequent examples.

Proposition 5. Let $k$ be a cardinal number less than or equal to $|\mathbf{R}|$.

(a) A transformation $Q$ belongs to $\mathcal{M}^{k}$ if and only if

$$
Q(S)=\bigcup_{S^{\prime} \subseteq S,\left|S^{\prime}\right| \leq k} Q\left(S^{\prime}\right)
$$

for every spatial database $S$.

(b) We have that

$$
\mathcal{M}^{0} \varsubsetneqq \mathcal{M}^{1} \varsubsetneqq \cdots \varsubsetneqq \mathcal{M}^{l} \varsubsetneqq \mathcal{M}^{l+1} \varsubsetneqq \cdots \varsubsetneqq \bigcup_{l \in \mathbf{N}} \mathcal{M}^{l} \varsubsetneqq \mathcal{M}^{|\mathbf{N}|} \varsubsetneqq \mathcal{M}^{|\mathbf{R}|}=\mathcal{M} .
$$


(c) If the transformations $Q_{1}$ and $Q_{2}$ belong to $\mathcal{M}^{k}, k \geq 0$, then so does $Q_{1} \cup Q_{2}$.

(d) The projection on a subspace $\mathbf{R}^{n} \subset \mathbf{R}^{m}$ belongs to $\mathcal{M}^{1}$. The selection of the points of a spatial database that belong to a fixed set $F \subset \mathbf{R}^{m}$ belongs to $\mathcal{M}^{1}$.

(e) If $Q_{1}$ and $Q_{2}$ belong to $\mathcal{M}$, then so does $Q_{1} \circ Q_{2}$. If $Q_{1}$ belongs to $\mathcal{M}^{1}$ and $Q_{2}$ belongs to $\mathcal{M}^{k}, k \geq 0$, the $Q_{1} \circ Q_{2}$ belongs to $\mathcal{M}^{k}$.

(f) There exist spatial transformations that are not monotone.

Example 1. 1. $\mathcal{M}^{0}$ is the set of spatial transformations that map every spatial database to some fixed figure in $\mathbf{R}^{m}$. These transformations obviously also belong to every $\mathcal{M}^{k}, k \geq 0$.

2. Translations, isometries, similarities, affinities of spatial databases belong to $\mathcal{M}^{1}$, and hence to every $\mathcal{M}^{k}, k \geq 1$.

3. "Return $\mathbf{R}^{m}$ if the diameter of the database is larger than 1, else return the empty set" belongs to $\mathcal{M}^{2}$ but not to $\mathcal{M}^{1}$.

4. The transformation that returns $\mathbf{R}^{m}$ on an input that contains at least $k$ points and the empty set in any other case, belongs to $\mathcal{M}^{k}$ but not to $\mathcal{M}^{k-1}$.

5. The transformation that maps a spatial database to its convex hull belongs to $\mathcal{M}^{m+1}$. Remark that here the degree depends on the dimension of the space $\mathbf{R}^{m}$ in which the database is embedded.

6. The transformation of a spatial database $S$ into the empty set if $|S|$ is finite and into $\mathbf{R}^{m}$ if $|S|$ is infinite belongs to $\mathcal{M}^{|\mathbf{N}|}$ but not to $\bigcup_{k \in \mathbf{N}} \mathcal{M}^{k}$.

7. The transformation of a spatial database to its topological interior, is a monotonic transformation, but not monotone of any finite degree nor of infinitely countable degree.

8. The complement transformation $Q(S)=S^{c}$ is not monotone of any degree.

\section{Sound and complete languages for monotonic transformations of finite degree}

In this section, we give sound and complete languages for the monotonic transformations that have a finite number of degrees of freedom and that are expressible in the spatial calculus.

Theorem 6. Let $k$ be a natural number and let $Q$ be a spatial database transformation that is expressible in the spatial calculus. The transformation $Q$ belongs to $\mathcal{M}^{k}$ if and only if $Q$ is equivalent to a finite union of monotonic transformations of degree $k$ that are expressible by conjunctive formulas of the form

$$
\bigwedge_{i=1}^{n} p_{i}(\mathbf{y}) \theta_{i} 0
$$

or

$$
\left(\exists \mathbf{x}_{1}\right) \cdots\left(\exists \mathbf{x}_{k}\right)\left(\bigwedge_{l=1}^{k} S\left(\mathbf{x}_{l}\right) \wedge \bigwedge_{i=1}^{n} p_{i}\left(\mathbf{y}, \mathbf{x}_{1}, \cdots, \mathbf{x}_{k}\right) \theta_{i} 0\right),
$$

where $p_{i}$ are polynomials with integer coefficients and $\theta_{i} \in\{\geq,>\}$. 
Proof. (Sketch) It is an easy set-theoretical exercise to show that the given conjunctive formulas express monotonic transformations of degree $k$. The ifdirection then follows immediately from (c) of Proposition 5.

For the only-if-direction, let $Q$ be a transformation expressed by the spatial calculus formula $\varphi$, i.e., $Q(S)=\{\mathbf{y} \mid \varphi(\mathbf{y}, S)\}$. We first remark that the equality

$$
Q(S)=\bigcup_{S^{\prime} \subseteq S,\left|S^{\prime}\right| \leq k} Q\left(S^{\prime}\right)
$$

implies that $Q(S)=Q(\emptyset)$ if $k=0$ and

$$
Q(S)=Q(\emptyset) \cup\left\{\mathbf{y} \mid\left(\exists \mathbf{x}_{1}\right) \cdots\left(\exists \mathbf{x}_{k}\right)\left(\bigwedge_{l=1}^{k} S\left(\mathbf{x}_{l}\right) \wedge \varphi\left(\mathbf{y},\left\{\mathbf{x}_{1}, \ldots, \mathbf{x}_{k}\right\}\right)\right)\right\}
$$

if $k>0$.

$Q(\emptyset)$ is a fixed $m$-dimensional spatial database and is therefore, as mentioned in Section 2, a Boolean combination of sets of the form $\{\mathbf{y} \mid p(\mathbf{y})>0\}$. This Boolean combination can be written as a union of intersections of the form

$$
\left\{\mathbf{y} \mid \bigwedge_{i=1}^{n} p_{i}(\mathbf{y}) \theta_{i} 0\right\}
$$

with $\theta_{i} \in\{\geq,>\}$.

For what concerns the other set, $\varphi\left(\mathbf{y},\left\{\mathbf{x}_{1}, \ldots, \mathbf{x}_{k}\right\}\right)$ is a spatial calculus formula with free variables $\mathbf{y}, \mathbf{x}_{1}, \ldots, \mathbf{x}_{k}$. Tarski's quantifier elimination property for the theory of the field of real numbers (see, e.g., $[6,8,22]$ ) guarantees the existence of a quantifier-free spatial calculus formula $\psi\left(\mathbf{y},\left\{\mathbf{x}_{1}, \ldots, \mathbf{x}_{k}\right\}\right)$ that is equivalent to $\varphi\left(\mathbf{y},\left\{\mathbf{x}_{1}, \ldots, \mathbf{x}_{k}\right\}\right)$. We can write $\psi\left(\mathbf{y},\left\{\mathbf{x}_{1}, \ldots, \mathbf{x}_{k}\right\}\right)$ in disjunctive normal form as

$$
\bigvee_{i=1}^{d} \bigwedge_{j=1}^{c_{i}} p_{i j}\left(\mathbf{y}, \mathbf{x}_{1}, \ldots, \mathbf{x}_{k}\right) \theta_{i j} 0
$$

with $p_{i j}$ polynomials with integers coefficients and $\theta_{i j} \in\{\geq,>\}$. Using some well-known logical equivalences we then obtain that

$Q(S)=Q(\emptyset) \cup\left\{\mathbf{y} \mid \bigvee_{i=1}^{d}\left(\left(\exists \mathbf{x}_{1}\right) \cdots\left(\exists \mathbf{x}_{k}\right)\left(\bigwedge_{l=1}^{k} S\left(\mathbf{x}_{l}\right) \wedge \bigwedge_{j=1}^{c_{i}} p_{i j}\left(\mathbf{y}, \mathbf{x}_{1}, \ldots, \mathbf{x}_{k}\right) \theta_{i j} 0\right)\right)\right\}$

This completes the proof.

To illustrate this result, we return to the convex hull. The convex hull of a spatial database $S$ is the smallest convex database that contains $S$. Usually, the convex hull of $S \subset \mathbf{R}^{m}$ is given as

$$
\begin{aligned}
&\left\{\mathbf{y} \mid\left(\exists \mathbf{x}_{1}\right) \cdots\left(\exists \mathbf{x}_{m+1}\right)\left(\exists \lambda_{1}\right) \cdots\left(\exists \lambda_{m+1}\right)\right.\left(\bigwedge_{i=1}^{m+1} S\left(\mathbf{x}_{i}\right) \wedge \bigwedge_{i=1}^{m+1} \lambda_{i} \geq 0\right. \\
&\left.\left.\wedge \lambda_{1}+\cdots+\lambda_{m+1}=1 \wedge \mathbf{y}=\lambda_{1} \mathbf{x}_{1}+\cdots+\lambda_{m+1} \mathbf{x}_{m+1}\right)\right\} .
\end{aligned}
$$


However, as mentioned before, the $m$-dimensional convex hull belongs to $\mathcal{M}^{m+1}$. Hence, the theorem tells us that it must be possible to express this with less quantifiers, namely by the quantifier prefix $\left(\exists \mathbf{x}_{1}\right) \cdots\left(\exists \mathbf{x}_{m+1}\right)$. Indeed, we can express the convex hull as

$$
\left\{\mathbf{y} \mid\left(\exists \mathbf{x}_{1}\right) \cdots\left(\exists \mathbf{x}_{m+1}\right)\left(\bigwedge_{i=1}^{m+1} S\left(\mathbf{x}_{i}\right) \wedge \mathrm{CH}\left(\mathbf{x}_{1}, \ldots, \mathbf{x}_{m+1}, \mathbf{y}\right)\right)\right\},
$$

where $\mathrm{CH}\left(\mathbf{x}_{1}, \ldots, \mathbf{x}_{m+1}, \mathbf{y}\right)$ abbreviates the formula that expresses that, for every subset of $m$ points from $\left\{\mathbf{x}_{1}, \ldots, \mathbf{x}_{m+1}\right\}$, the point $\mathbf{y}$ lies in the appropriate halfspace determined by that subset. This formula can be expressed without the use of quantifiers. The convex hull of a 1-dimensional database $S$ is, e.g., given by

$$
\left\{y \mid\left(\exists x_{1}\right)\left(\exists x_{2}\right)\left(S\left(x_{1}\right) \wedge S\left(x_{2}\right) \wedge x_{1} \leq y \wedge y \leq x_{2}\right)\right\}
$$

The previous example illustrates how Theorem 6 can be used to write certain transformations in a more compact way. The main importance of Theorem 6 , however, is that it provides a syntactic framework, by means of a normal form for calculus expressions, in which transformations are guaranteed to belong to $\mathcal{M}^{k}$. In other words, for every finite number $k$, Theorem 6 provides for a sound and complete language in which only transformations that belong to $\mathcal{M}^{k}$ can be written, but in which also all transformations of $\mathcal{M}^{k}$ expressible in the calculus, can be written.

\section{Monotonic transformations of infinite degree}

In this section, we search for sound and complete languages for monotonic transformations of infinite degree that are expressible in the spatial calculus.

It might seem that, since the spatial calculus is incapable of characterizing infinitely countable sets, there are no differences between different infinite degrees of monotonicity for transformations expressible in the calculus. The following proposition shows that this is not the case.

Proposition 7. For transformations expressible in the spatial calculus, the following inclusions are strict:

$$
\bigcup_{k \in \mathbf{N}} \mathcal{M}^{k} \varsubsetneqq \mathcal{M}^{|\mathbf{N}|} \varsubsetneqq \mathcal{M}^{|\mathbf{R}|}=\mathcal{M} .
$$

Clearly, there exist also transformations expressible in the spatial calculus that are not monotone.

Proof. The transformations in 6 and 7 of Example 1 are expressible in the calculus (see [20]). This proves the strictness of the two inclusions. The last transformation of Example 1 is expressible in the calculus. This proves the second claim. 
Obviously, the union over $\mathbf{N}$ of the sound and complete languages for $\mathcal{M}^{k}$, as provided by Theorem 6 , yields a sound and complete language for the spatial calculus transformations that belong to $\bigcup_{k \in \mathbf{N}} \mathcal{M}^{k}$. We therefore have

Theorem 8. Let $Q$ be a transformation that is expressible in the spatial calculus. The transformation $Q$ belongs to $\bigcup_{k \in \mathbf{N}} \mathcal{M}^{k}$ if and only if $Q$ is equivalent to a finite union of monotonic transformations of finite degree that are expressible by conjunctive formulas of the form

$$
\bigwedge_{i=1}^{n} p_{i}(\mathbf{y}) \theta_{i} 0
$$

or

$$
\left(\exists \mathbf{x}_{1}\right) \cdots\left(\exists \mathbf{x}_{k}\right)\left(\bigwedge_{l=1}^{k} S\left(\mathbf{x}_{l}\right) \wedge \bigwedge_{i=1}^{n} p_{i}\left(\mathbf{y}, \mathbf{x}_{1}, \cdots, \mathbf{x}_{k}\right) \theta_{i} 0\right),
$$

for some $k \in \mathbf{N} \backslash\{0\}$ and with $p_{i}$ polynomials with integer coefficients and $\theta_{i} \in\{\geq,>\}$.

We now turn our attention to $\mathcal{M}^{|\mathbf{N}|}$ and $\mathcal{M}^{|\mathbf{R}|}$. Here, the story is more complicated. From model theory, we know that first-order logic sentences that are preserved under homomorphisms, i.e., that are monotone, have a "positive" equivalent. This property is usually referred to as Lyndon's homomorphism theorem $[3,14]$. Thus, it is a natural question to ask whether also every monotonic spatial transformation is expressible by a spatial calculus formula that is positive. More technically, we call a spatial calculus formula positive if every atomic subformula $S\left(y_{1}, \ldots, y_{m}\right)$ lies within the scope of an even number of negation symbols. We can easily prove by induction on the length of formulas that

Proposition 9. A transformation that is expressed by a positive spatial calculus formula is monotone.

We can use the transformation of a spatial database into its topological interior as an illustration. Indeed, for 2-dimensional databases, for example, this transformation can be expressed by the positive formula

$$
(\exists \varepsilon)\left(\forall x^{\prime}\right)\left(\forall y^{\prime}\right)\left(\varepsilon>0 \wedge\left(\left(x-x^{\prime}\right)^{2}+\left(y-y^{\prime}\right)^{2} \geq \varepsilon \vee S\left(x^{\prime}, y^{\prime}\right)\right)\right) .
$$

Hence, it is monotone (as already observed before).

The converse of Proposition 9, on the other hand, is not obvious. Ajtai and Gurevich have shown that Lyndon's theorem fails when only finite models are considered [2].

We will show that Lyndon's theorem also fails in our setting:

Theorem 10. There is a monotonic spatial transformation, expressible in the spatial calculus, that is not expressible by a positive spatial calculus formula.

For a sketch of the proof we refer to the Appendix.

We conclude this section with one positive result: 
Proposition 11. A monotonic spatial transformation that is expressible by a quantifier-free spatial calculus formula is positively expressible in the spatial calculus.

Proof. (Sketch) We first give the proof for 1-dimensional spatial transformations. Then we sketch how it can be generalized to higher dimensional transformations.

So, let $Q(S)=\{x \mid \varphi(x, S)\}$ be a 1-dimensional spatial transformation. $\varphi(x, S)$ can be written in disjunctive normal form as

$$
\left(S(x) \wedge \pi_{1}(x)\right) \vee\left(\neg S(x) \wedge \pi_{2}(x)\right),
$$

where $\pi_{1}(x)$ and $\pi_{2}(x)$ are Boolean combinations of polynomial inequalities. We can write this formula also as $\varphi_{1}(x, S) \vee \varphi_{2}(x, S) \vee \varphi_{3}(x, S)$, with

$$
\begin{aligned}
& \varphi_{1}(x, S)=S(x) \wedge \pi_{1}(x) \wedge \neg \pi_{2}(x), \\
& \varphi_{2}(x, S)=\neg S(x) \wedge \pi_{2}(x) \wedge \neg \pi_{1}(x), \text { and } \\
& \varphi_{3}(x, S)=\pi_{1}(x) \wedge \pi_{2}(x) .
\end{aligned}
$$

We now consider two cases. If $\varphi_{2}(x, S)$ is false for all $x \in \mathbf{R}$ and all spatial databases $S$, then $\varphi(x, S)$ is equivalent to $\varphi_{1}(x, S) \vee \varphi_{3}(x, S)$, and therefore positively expressible.

If, on the other hand, there exists a spatial database $S_{0}$ and $x_{0} \in \mathbf{R}$, such that $\varphi_{2}\left(x_{0}, S_{0}\right)$ is true, we can easily show that $x_{0} \in Q\left(S_{0}\right)$, but $x_{0} \notin Q\left(S_{0} \cup\left\{x_{0}\right\}\right)$. Therefore, $Q$ is not monotone. Thus the second case cannot occur. This completes the proof for the 1-dimensional case.

For the $m$-dimensional case, we also can write any quantifier-free formula $\varphi\left(x_{1}, \ldots, x_{n}, S\right)$ in disjunctive normal form with $2^{\left(m^{m}\right)}$ disjuncts, each of which is a conjunction of $m^{m}$ conjuncts of the form $S\left(y_{1}, \ldots, y_{n}\right)$ or $\neg S\left(y_{1}, \ldots, y_{n}\right)$, with $y_{i} \in\left\{x_{1}, \ldots x_{n}\right\}$, and of a conjunct $\pi\left(x_{1}, \ldots x_{n}\right)$, which is a Boolean combination of polynomial inequalities. As in the 1-dimensional case, we can then split up the polynomial parts which results in additional disjuncts consisting of $\mathrm{m}^{m}$, $m^{m}-1, \ldots$ conjuncts that are disjoint with the longer disjuncts. We can then apply an argumentation, similar to the 1-dimensional case, that shows that no negation can occur.

\section{$5 \quad$ Undecidability and decidability results}

In this section, we show that it is undecidable whether a spatial transformation expressible in the spatial calculus belongs to $\mathcal{M}^{k}$. We also show that many interesting properties of spatial transformations that are undecidable in general (see [20]) become decidable for monotonic transformations of finite degree, in contrast to our first result.

Theorem 12. The following problems are undecidable: 
(a) Given a spatial transformation $Q$, tell whether $Q$ is monotone, and

(b) For $k \in \mathbf{N}$ and a given spatial transformation $Q$, tell whether $Q$ belongs to $\mathcal{M}^{k}$.

Proof. (Sketch) Statement (a) follows directly from the fact that it is undecidable whether a first-order sentence $\varphi(S)$ is monotone [12].

For (b) and $k=0$, we observe that two spatial transformations $Q_{1}(S)=$ $\left\{\mathbf{y} \mid \varphi_{1}(\mathbf{y}, S)\right\}$ and $Q_{2}(S)=\left\{\mathbf{y} \mid \varphi_{2}(\mathbf{y}, S)\right\}$ are equivalent if and only if $Q(S)=$ $\left\{\mathbf{y} \mid \varphi_{1}(\mathbf{y}, S) \wedge \neg \varphi_{2}(\mathbf{y}, S)\right\}$ belongs to $\mathcal{M}^{0}$ and $Q(\emptyset)=\emptyset$. The latter condition is decidable. Since equivalence of spatial transformations is undecidable [20], it follows that membership of $\mathcal{M}^{0}$ is undecidable.

To prove the theorem for $k \in \mathbf{N} \backslash\{0\}$, we can use a reduction technique that was introduced in [20]. We sketch the proof for $k=1$ in dimension 1. Other cases can be proved analogously.

It is well-known that the $\exists^{*}$-fragment of number theory is undecidable. Encode a natural number $n$ by the 1-dimensional spatial database

$$
\operatorname{enc}(n)=\{0,1, \ldots, n\}
$$

and encode a vector of natural numbers $\left(n_{1}, \ldots, n_{k}\right)$ by the database

$\operatorname{enc}\left(n_{1}, \ldots, n_{k}\right)=\operatorname{enc}\left(n_{1}\right) \cup\left(\operatorname{enc}\left(n_{2}\right)+n_{1}+2\right) \cup \cdots \cup\left(\operatorname{enc}\left(n_{k}\right)+n_{1}+2+\cdots+n_{k-1}+2\right)$.

The corresponding decoding is expressible in the spatial calculus. We can reduce the decision of the truth of a $\exists^{*}$-sentence $(\exists \mathbf{x}) \psi(\mathbf{x})$ of number theory to deciding whether the transformation "if $S$ encodes $\mathbf{x}$ and $\psi(\mathbf{x})$, return $\mathbf{R}$, else return the empty set" belongs to $\mathcal{M}^{1}$.

Many interesting properties of spatial transformations, such as equivalence of transformations, containment, and genericity are undecidable for transformations expressed in the spatial calculus (see [20]). For monotonic transformations of finite degree, many of these properties become decidable. As an illustration, we give

Theorem 13. Let $k$ be a natural number. Let $Q, Q_{1}$ and $Q_{2}$ be spatial transformations that are expressible in the spatial calculus and that belong to $\mathcal{M}^{k}$. The following properties are decidable:

- $Q_{1}$ and $Q_{2}$ are equivalent,

- $Q_{1}$ is contained in $Q_{2}$,

- $Q$ is generic,

- for every $S, Q(S)$ contains a line.

Proof. (Sketch) As an example, we give the proof for containment. Suppose that $Q_{1}$ and $Q_{2}$ are expressed by spatial calculus formulas $\varphi_{1}$ and $\varphi_{2} . Q_{1}$ is contained in $Q_{2}$ if for all databases $S, Q_{1}(S) \subseteq Q_{2}(S)$ holds. If $Q_{1}$ and $Q_{2}$ are in $\mathcal{M}^{k}$, this second-order condition is equivalent to $Q_{1}(\emptyset) \subseteq Q_{2}(\emptyset)$ and

$$
\left(\forall \mathbf{x}_{1}\right) \cdots\left(\forall \mathbf{x}_{k}\right)(\forall \mathbf{y})\left(\varphi_{1}\left(\mathbf{y},\left\{\mathbf{x}_{1}, \ldots \mathbf{x}_{k}\right\}\right) \rightarrow \varphi_{2}\left(\mathbf{y},\left\{\mathbf{x}_{1}, \ldots \mathbf{x}_{k}\right\}\right)\right) .
$$


Both are first-order sentences in the theory of the field of real numbers. From Tarski's quantifier elimination property it follows that this sentence can be decided (see, e.g., $[6,8,22]$ ).

\section{Discussion}

We end this paper with the discussion of two topics. First, we address the question of how unique the hierarchy described in this paper is. Secondly, we state some open problems concerning sound and complete languages for the class of all monotonic transformations.

\subsection{Alternative hierarchies of monotonicity classes.}

In this paper, we have discussed a hierarchy of monotonicity classes of spatial transformations that is indexed by the degrees of freedom of spatial transformations, i.e., that is indexed by cardinal numbers. In our opinion, this hierarchy is a very natural one. It is, however, not the only possible one.

In the hierarchy proposed in this paper, the monotonic transformations have the form

$$
Q(S)=\bigcup_{S^{\prime} \subseteq S,\left|S^{\prime}\right| \leq k} Q\left(S^{\prime}\right)
$$

where $k$ is (in most cases) finite, i.e., the transformation is determined by its images on certain finite subsets.

However, we can also consider a hierarchy of monotonic transformations that are determined by their image on certain infinite subsets. We then obtain a hierarchy indexed by classes of infinite figures. We give an example of this and restrict our attention for a moment to the plane $\mathbf{R}^{2}$. Take, for instance, $\mathcal{P}_{n}$ to be the class of figures that are the convex hull of regular polygons of $i$ sides with $i \leq n$. Let $\mathcal{N}^{n}$ be the class of transformations of the form

$$
Q(S)=\bigcup_{S^{\prime} \subseteq S, S^{\prime} \in \mathcal{P}_{n}} Q\left(S^{\prime}\right)
$$

The elements of $\mathcal{N}^{n}$ are also monotone. They also form a hierarchy of monotonicity classes for increasing $n: \mathcal{N}^{0} \varsubsetneqq \mathcal{N}^{1} \varsubsetneqq \mathcal{N}^{2} \varsubsetneqq \cdots$.

For each $n \in \mathbf{N}$, we also obtain sound and complete languages, in the style of Theorem 6 , that express exactly the transformations in $\mathcal{N}^{n}$ that are expressible in the spatial calculus. Indeed, in Theorem 6 , we needed a finite number of quantifiers to quantify over subsets of the database of some finite cardinality. To quantify over polygonal subsets of the database, also a finite number of quantifiers suffice.

More results in the style of Theorem 6 are possible. We give one more example, interesting in its own. For transformations in $\mathbf{R}^{m}$, consider the class of monotonic spatial transformations that satisfy

$$
Q(S)=\bigcup_{S^{\prime} \subseteq S, S^{\prime} \in \mathcal{B}_{m}} Q\left(S^{\prime}\right),
$$


where $\mathcal{B}_{m}$ is the class of $m$-dimensional open spheres with a radius $r \geq 0$. The transformation of a spatial database into its topological interior belongs to this class. Again, in the style of Theorem 6, we obtain that spatial transformations expressible in the spatial calculus belong to this class if and only if they are expressible as a finite union of spatial transformations expressible by formulas of the form

or

$$
\bigwedge_{i=1}^{n} p_{i}(\mathbf{y}) \theta_{i} 0
$$

$$
(\exists \mathbf{x})(\exists \varepsilon>0)\left((\forall \mathbf{z})\left(\mathrm{d}_{m}(\mathbf{x}, \mathbf{z})<\varepsilon \rightarrow S(\mathbf{z})\right) \wedge \bigwedge_{i=1}^{n} p_{i}(\mathbf{x}, \mathbf{y}, \varepsilon) \theta_{i} 0\right),
$$

where $p_{i}$ are polynomials with integer coefficients and $\theta_{i} \in\{\geq,>\}$, where $\mathrm{d}_{m}(\mathbf{x}, \mathbf{z})$ abbreviates the quadratic polynomial $\sum_{i=1}^{m}\left(x_{i}-z_{i}\right)^{2}$, and where $\mathbf{y}$ are the result vectors.

All the above formulas are positive in $S$. This proves, in an alternative way, that the transformation of a spatial database into its topological interior is monotone.

The examples given in this section show that other hierarchies are possible. Depending on the applications one has in mind one may be preferred to the other.

\subsection{Is there a sound and complete language for all monotonic spatial calculus transformations?}

In Section 4, we have searched for a sound and complete language for all spatial transformations expressible in the spatial calculus. The only likely candidate for such a language seems to be the set of positive spatial calculus formulas. Since Theorem 10 rules out this possibility, the search for a complete language seems a very difficult, if not impossible task.

The same problem remains for $\mathcal{M}^{|\mathbf{N}|}$. We therefore state the following:

Open problem 14. Is there a sound and complete language that captures the transformations expressible in the spatial calculus

- that belong to $\mathcal{M}^{|\mathbf{R}|}$ ?

- that belong to $\mathcal{M}^{|\mathbf{N}|}$ ?

The only positive result we can show in this context is that Lyndoms' theorem holds when the attention is restricted to spatial transformations expressible by a quantifier-free formula (see Proposition 11). Gurevich points out a number of other classes of first-order sentences for which Lyndon's theorem survives in the case of finite structures [12]. He mentions existential sentences, universal sentences, prenex sentences with prefix $\exists^{n} \forall$, and prenex sentences with prefix $\forall^{n} \exists$. Also in the context of the spatial calculus, these languages seem interesting candidates for further research.

Another interesting question is the following: although for spatial databases monotonic formulas cannot be translated into positive formulas, do the least fixed points of these two classes coincide? 


\section{Acknowledgments}

The author is indebted to Jan Paredaens and Jan Van den Bussche for the collaborative research that has lead to several of the results discussed in this paper and to Stijn Dekeyser for proofreading earlier versions of the paper. The author also wishes to thank Marc Gyssens whose comments considerably improved the presentation of the paper.

\section{References}

1. D. Abel and B.C. Ooi, editors. Advances in spatial databases-3rd Symposium SSD'93, volume 692 of Lecture Notes in Computer Science, Springer-Verlag, 1993.

2. M. Ajtai and Y. Gurevich. Monotone versus Positive. Journal of the Association for Computing Machinery, Vol. 34, pages 1004-1015, October 1987.

3. J. Barwise, editor. Handbook of Mathematical Logic. North-Holland Publishing Company, Amsterdam, 1989.

4. M. Benedikt, G. Dong, L. Libkin, and L. Wong. Relational expressive power of constraint query languages. In Proceedings 15th ACM Symposium on Principles of Database Systems (PODS'96), pages 1-16, ACM Press, 1996.

5. M. Benedikt and L. Libkin. Languages for Relational Databases over Interpreted Structures. In Proceedings 16th ACM Symposium on Principles of Database Systems (PODS'97), pages 87-98, ACM Press, 1997.

6. J. Bochnak, M. Coste, and M.-F. Roy. Géométrie algébrique réelle. SpringerVerlag, 1987.

7. A. Buchmann, editor. Design and implementation of large spatial databases-First Symposium SSD'89, volume 409 of Lecture Notes in Computer Science. SpringerVerlag, 1989.

8. G.E. Collins. Quantifier elimination for real closed fields by cylindrical algebraic decomposition. In Automata theory and formal languages, volume 33 of Lecture Notes in Computer Science, pages 134-183, Springer-Verlag, 1975.

9. M. Coste. Ensembles semi-algébriques. In Géometrie algébrique réelle et formes quadratiques, volume 959 of Lecture Notes in Mathematics, pages 109-138, Springer-Verlag, 1982.

10. M.J. Egenhofer and J.R. Herring, editors. Advances in Spatial Databases, volume 951 of Lecture Notes in Computer Science, Springer-Verlag, 1995.

11. O. Gunther and H.-J. Schek, editors. Advances in spatial databases - 2nd Symposium SSD'91, volume 525 of Lecture Notes in Computer Science. Springer-Verlag, 1991.

12. Y. Gurevich. Toward logic tailored for computational complexity. In Computation and Proof Theory, M. Richter et al., editors, volume 1104 of Lecture Notes in Mathematics, pages 175-216, Springer, 1984.

13. M. Gyssens, J. Van den Bussche, and D. Van Gucht. Complete geometrical query languages. In Proceedings of the 16th ACM Symposium on Principles of Database Systems (PODS'97), pages 62-67, ACM Press, 1997.

14. W. Hodges. Model Theory. Cambridge University press, Cambridge, 1995.

15. P.C. Kanellakis, G.M. Kuper, and P.Z. Revesz. Constraint query languages. Journal of Computer and System Sciences, 51(1):26-52, August 1995. 
16. B. Kuijpers, J. Paredaens, M. Smits, and J. Van den Bussche. Termination properties of spatial Datalog programs. In Proceedings of "Logic in Databases" (LID'96), D. Pedreschi and C. Zaniolo, editors, volume 1154 of Lecture Notes in Computer Science, pages 101-116, Springer-Verlag, 1996.

17. B. Kuijpers and M. Smits. On expressing topological connectivity in spatial Datalog. In Proceedings of the workshop Constraint Databases and Their Applications (CDB'97), V. Gaede, A. Brodsky, O. Gunter, D. Srivastava, V. Vianu, M. Wallace, editors, volume 1191 of Lecture Notes in Computer Science, pages 116-133, Springer-Verlag, 1997.

18. G. McColm. The dimension of the Negation of Transitive Closure. The Journal of Symbolic Logic, Vol. 60, Nr. 2, pages 392-414, June 1995.

19. L. Libkin. Personal communication. August 1997.

20. J. Paredaens, J. Van den Bussche, and D. Van Gucht. Towards a theory of spatial database queries. In Proceedings of the 13th ACM Symposium on Principles of Database Systems (PODS'94), pages 279-28, ACM Press, 1994.

21. A. P. Stolboushkin. Finitely Monotone Properties. In Proceedings of the Tenth Annual IEEE Symposium on Logic in Computer Science (LICS'95), pages 324330, IEEE Computer Society Press, 1995.

22. A. Tarski. A Decision Method for Elementary Algebra and Geometry. University of California Press, 1951.

\section{Appendix}

In the following pages we sketch the proof of Theorem 10. Our proof is inspired by an alternative proof of the result of Ajtai and Gurevich that was given by Stolboushkin [21]. It uses a characterization of definability by positive first-order formulas by means of an Ehrenfeucht-Fraïssé-like game. This characterization was developed by Stolboushkin [21] and, independently, by McColm [18].

The spatial transformation $Q$ that witnesses Theorem 10 actually is a transformation that works on two 2-dimensional spatial databases $S$ and $T$. The transformation $Q$ is expressed by a spatial calculus sentence $\varphi(S, T)$. This means that viewed as a spatial transformation, $Q$ transforms the pair $(S, T)$ either into $\emptyset$ or into $\mathbf{R}^{2}$. We now give a detailed description of $Q(S, T)$.

Let $\psi(S, T, y)$ be the formula $(\forall x)((T(x, y) \vee x=y) \rightarrow(\forall z)(S(z, x) \rightarrow$ $(\forall u)((T(u, z) \vee u=z) \rightarrow(T(u, x) \wedge T(u, y)))))$.

Then $\varphi(S, T)$ is equivalent to the sentence

$\varphi_{0}(S, T) \vee \varphi_{1}(S, T) \vee \varphi_{2}(S, T) \vee \varphi_{3}(S, T) \vee \varphi_{4}(S, T) \vee\left(\varphi_{5}(S, T) \wedge \varphi_{6}(S, T)\right)$,

with 


$$
\begin{aligned}
& \varphi_{0}(S, T)= \text { } S \text { is infinite" } \vee \text { " } T \text { is infinite"; } \\
& \varphi_{1}(S, T)=(\exists x)(\exists y)(S(x, y) \wedge S(y, x)) ; \\
& \varphi_{2}(S, T)=(\exists x)(\exists y)(T(x, y) \wedge T(y, x)) ; \\
& \varphi_{3}(S, T)=(\exists x)(\exists y)((\forall z)(\neg S(z, y) \wedge T(x, y)) ; \\
& \varphi_{4}(S, T)=(\exists x)(\exists z)((\forall y)(S(y, x) \rightarrow \psi(S, T, y)) \wedge T(z, x) \wedge(\forall y)(S(y, x) \rightarrow \\
&\quad(\neg(T(z, y) \vee z=y))) ; \\
& \varphi_{5}(S, T)=(\forall x)(\forall y)(S(x, y) \rightarrow T(x, y)) ; \text { and finally } \\
& \varphi_{6}(S, T)=(\forall x)(\forall y)(\forall z)(T(x, y) \wedge T(y, z) \rightarrow T(x, z)) .
\end{aligned}
$$

We remark that $\varphi_{0}(S, T)$ is expressible in the spatial calculus. For instance, " $S$ is infinite" is expressed by the sentence $\neg(\forall x)(\forall y)\left(S(x, y) \rightarrow\left((\exists \varepsilon>0)\left(\forall x^{\prime}\right)\right.\right.$ $\left.\left(\forall y^{\prime}\right)\left(\left(\left(x-x^{\prime}\right)^{2}+(y-y)^{2}<\varepsilon \wedge S\left(x^{\prime}, y^{\prime}\right)\right) \rightarrow\left(x=x^{\prime} \wedge y=y^{\prime}\right)\right)\right)$. For finite $S$ and $T, \varphi_{1}(S, T) \vee \varphi_{2}(S, T) \vee \varphi_{3}(S, T) \vee \varphi_{4}(S, T) \vee\left(\varphi_{5}(S, T) \wedge \varphi_{6}(S, T)\right)$ characterizes a class of structures that contains what Stolboushkin calls grids [21]. This sentence, however returns false on incomplete grids.

We now first show that $Q(S, T)=\left\{(x, y) \in \mathbf{R}^{2} \mid \varphi(S, T)\right\}$ is monotone in $T$, i.e., that $T_{1} \subseteq T_{2}$ implies that $Q\left(S, T_{1}\right) \subseteq Q\left(S, T_{2}\right)$.

Lemma 15. The transformation $Q(S, T)$ is monotone in $T$.

Proof. For finite $S$ and $T$ the lemma was proven in [21] (Lemma 4.4). For $S$ or $T$ infinite, $\varphi_{0}(S, T)$ guarantees monotonicity.

In order to formulate the next results, we turn to some more precise terminology from logic. The transformation $Q(S, T)$, as given above, is expressed by a first-order sentence over the signature $\langle S, T,+, \times,<, 0,1\rangle$. This sentence is, however, invariant under monotonic transformations of $\mathbf{R}$. From a collapse theorem on the expressiveness of first-order logic of the reals by Benedikt, Dong, Libkib, and Wong [4] (see also [5]) it follows that

Lemma 16. The transformation $Q(S, T)$ can be expressed by a first-order sentence $\bar{\varphi}(S, T,<)$ over the signature $\langle S, T,<\rangle$.

We will now describe how a characterization of definability by positive firstorder sentences by means of an Ehrenfeucht-Fraïssé-like game will lead us to the conclusion that $Q(S, T)$ is not expressible by a formula that is positive in $T .^{3}$ This characterization and these games are described in [18] and [21]. First, we will describe the games specialized to our situation (i.e., specialized to the

${ }^{3}$ A formula is positive in $T$ if every subformula of the form $T(u, v)$ is in the scope of an even number of negation symbols. 
signature $\langle S, T,<\rangle)$. Next, we will specialize the characterization theorem of [18] and [21] to our setting.

The pebble game is played by two players, the Spoiler and the Duplicator, on two $\langle S, T,<\rangle$-structures $\mathcal{A}=\left(S^{A}, T^{A},<^{A}\right)$ and $\mathcal{B}=\left(S^{B}, T^{B},<^{B}\right)$. The underlying domain of these structures is $\mathbf{R}$. As usual, the Spoiler tries to distinguish between these structures, while the Duplicator tries to show that they are equivalent.

The game, which we will call the positive game from $\mathcal{A}$ to $\mathcal{B}$ of depth $n$, consists of $n$ rounds in which $n$ pebbles will be placed on elements $a_{1}, \ldots, a_{n}$ in $\mathcal{A}$ and $n$ pebbles will be placed on elements $b_{1}, \ldots, b_{n}$ in $\mathcal{B}$. The $i$ th round of the game consists of the Spoiler choosing one of the structures $\mathcal{A}$ and $\mathcal{B}$, placing a pebble on the domain of that structure, and the Duplicator placing a pebble in the domain of the other structure. After $n$ rounds, for each round $i(i \leq n)$, there are pebbles on $a_{i}$ in $\mathcal{A}$ and on $b_{i}$ in $\mathcal{B}$ respectively.

We say that the Duplicator wins the positive game from $\mathcal{A}$ to $\mathcal{B}$ of depth $n$ if and only if after the $n$ moves of the game the following four conditions are fulfilled

1. for all $i, j \leq n, a_{i}=a_{j}$ if and only if $b_{i}=b_{j}$;

2. for all $i, j \leq n, a_{i}<a_{j}$ if and only if $b_{i}<b_{j}$;

3. for all $i, j \leq n, S\left(a_{i}, a_{j}\right)$ if and only if $S\left(b_{i}, b_{j}\right)$;

4. for all $i, j \leq n, T\left(a_{i}, a_{j}\right)$ implies $T\left(b_{i}, b_{j}\right)$.

The following lemma is due to Stolboushkin [21] and McColm [18]. The converse is also in [21].

Lemma 17. Let $\chi(S, T,<)$ be a first-order sentence of quantifier depth $n$ over the signature $\langle S, T,<\rangle$ that is positive in $T$. If the Duplicator has a winning strategy for the positive game from structure $\mathcal{A}=\left(S^{A}, T^{A},<^{A}\right)$ to structure $\mathcal{B}=\left(S^{B}, T^{B},<^{B}\right)$ of depth $n$, then $\mathcal{A} \models \chi(S, T,<)$ implies that $\mathcal{B} \models \chi(S, T,<)$.

We will now describe two $\langle S, T,<\rangle$-structures $\mathcal{A}=\left(S^{A}, T^{A},<^{A}\right)$ and $\mathcal{B}=$ $\left(S^{B}, T^{B},<^{B}\right)$ such that the Duplicator has a winning strategy for the positive $n$-round game from $\mathcal{A}$ to $\mathcal{B}$. These structures are inspired by the finite grid structures described by Stolboushkin [21]. As mentioned before, the domain of both structures is $\mathbf{R}$. Both $<^{A}$ and $<^{B}$ are the natural order on the real line. $S^{A}$ is the finite relation

$$
\begin{gathered}
\left\{\left(i \cdot 2^{n+2}+j, i \cdot 2^{n+2}+j+1\right) \mid 0 \leq i<2^{n+1} \text { and } 1 \leq j<2^{n+2}\right\} \cup \\
\left\{\left(i \cdot 2^{n+2}+j,(i+1) \cdot 2^{n+2}+j\right) \mid 0 \leq i<2^{n+1}-1 \text { and } 1 \leq j \leq 2^{n+2}\right\}
\end{gathered}
$$

and $T^{A}$ is the transitive closure of $S^{A} . S^{B}$ is the same finite relation as $S^{A}$ and $T^{B}=T^{A} \backslash\left\{\left(2^{n+1}, 2^{n+1} \cdot\left(2^{n+2}-1\right)\right)\right\}$.

We have the following lemma:

Lemma 18. The Duplicator has a winning strategy for the positive n-round game from $\mathcal{A}$ to $\mathcal{B}$. 
Proof. (Sketch) We omit the details of the proof, but point out that the structures $\mathcal{A}=\left(S^{A}, T^{A},<^{A}\right)$ and $\mathcal{B}=\left(S^{B}, T^{B},<^{B}\right)$ correspond for what concerns their $S$ and $T$ components to what Stolboushkin calls a grid and a reduced grid. However, these grids are placed in a particular way on the real line: the different rows of Stolboushkin's grids are placed one after the other on $\mathbf{R}$. The game strategy outlined in [21] can then be adapted to fit this situation. An additional complication is that the game in [21] is played on finite structures while our game is played on an infinite structure.

The next lemma follows directly from [21].

Lemma 19. Let $\mathcal{A}$ and $\mathcal{B}$ be the structures described above. Let $\bar{\varphi}(S, T,<)$ be the first-order sentence that expresses $Q(S, T)$. Then $\mathcal{A} \models \bar{\varphi}(S, T,<)$ and $\mathcal{B} \not \models$ $\bar{\varphi}(S, T,<)$.

This proves that $\bar{\varphi}(S, T,<)$ is not positively expressible in $T$ over the signature $\langle S, T,<\rangle$. Indeed, if $\bar{\varphi}(S, T,<)$ would be positively expressible in $T$, then, by Lemma 17, it should also evaluate to true on $\mathcal{B}$, which, according to Lemma 19, it does not.

To complete the proof of Theorem 10, it suffices to prove that

Lemma 20. The formula $\bar{\varphi}(S, T,<)$ is not positively expressible in $T$ over the signature $\langle S, T,+, \times,<, 0,1\rangle$.

Proof. (Sketch) Suppose that $\bar{\varphi}(S, T,<)$ is positively expressible in $T$ over $\langle S, T,+, \times,<, 0,1\rangle$, say by $\psi(S, T,+, \times,<, 0,1)$. The formula $\psi(S, T,+, \times,<, 0,1)$ is equivalent to " $S$ is infinite" $\vee$ " $T$ is infinite" $\vee \bar{\psi}(S, T,+, \times,<, 0,1)$, where the sentence $\bar{\psi}(S, T,+, \times,<, 0,1)$ is $\psi(S, T,+, \times,<, 0,1)$ but considered to work on finite databases. $\bar{\psi}(S, T,+, \times,<, 0,1)$ is generic and therefore expressible over the signature $\langle S, T,<\rangle[5]$. Libkin [19] has shown that it follows from results in [5] that $\bar{\psi}(0,1,+, \times,<, 0,1)$ is also positively expressible in $T$ over the signature $\langle S, T,<\rangle$. Since " $S$ is infinite" $\vee$ " $T$ is infinite" can also be expressed in a positive way over $\langle S, T,<\rangle$, we obtain a contradiction. 